\title{
Assembly of Colloidal Semiconductor Nanorods in Solution by Depletion Attraction
}

\author{
Dmitry Baranov, ${ }^{\dagger, \perp}$ Angela Fiore, ${ }^{\dagger,}{ }^{\ddagger}$ Marijn van Huis, ${ }^{\S}$ Cinzia Giannini, ${ }^{\prime}$ Andrea Falqui, ${ }^{\dagger}$ \\ Ugo Lafont, ${ }^{\S}$ Henny Zandbergen, ${ }^{\S}$ Marco Zanella, ${ }^{\dagger}$ Roberto Cingolani, ${ }^{\dagger,}{ }^{\ddagger}$ and \\ Liberato Manna* ${ }^{*}$
}

$\dagger$ Istituto Italiano di Tecnologia, Via Morego 30, 16163 Genova, Italy, ${ }^{\dagger}$ National Nanotechnology Laboratory, Via per Arnesano, 73100, Lecce, Italy, ${ }^{\S}$ Kavli Institute of Nanoscience, Delft University of Technology, Lorentzweg 1 , NL-2628 CJ Delft, The Netherlands, and "CNR-Istituto di Crystallografia (IC), Via Amendola 122/O, I-70126, Bari, Italy

\begin{abstract}
Arranging anisotropic nanoparticles into ordered assemblies remains a challenging quest requiring innovative and ingenuous approaches. The variety of interactions present in colloidal solutions of nonspherical inorganic nanocrystals can be exploited for this purpose. By tuning depletion attraction forces between hydrophobic colloidal nanorods of semiconductors, dispersed in an organic solvent, these could be assembled into 2D monolayers of close-packed hexagonally ordered arrays directly in solution. Once formed, these layers could be fished onto a substrate, and sheets of vertically standing rods were fabricated, with no additional external bias applied. Alternatively, the assemblies could be isolated and redispersed in polar solvents, yielding suspensions of micrometersized sheets which could be chemically treated directly in solution. Depletion attraction forces were also effective in the shape-selective separation of nanorods from binary mixtures of rods and spheres. The reported procedures have the potential to enable powerful and cost-effective fabrication approaches to materials and devices based on self-organized anisotropic nanoparticles.
\end{abstract}

KEYWORDS Nanorods, nanocrystals, self-assembly, depletion forces

$\mathrm{T}$ he fabrication of ordered assemblies from nanoscale building blocks over large areas and volumes is of great interest in nanotechnology, as it represents a sustainable pathway toward the engineering of new materials and devices. In the spatial organization of nanoscale objects, many types of interactions can play a role,${ }^{1-3}$ although a deep understanding and handling of these interactions are far from being achieved. Despite recent successes in self-organization of nearly monodisperse spherical colloidal nanoparticles, ${ }^{4}$ self-assembly of shape controlled nanocrystals is much more difficult to achieve. ${ }^{5}$ Consequently, long-range assembly of anisotropic nanocrystals has proven to be more difficult by controlled evaporation alone than for their spherical counterparts. This is unfortunate, since most of the promises from assembly of nanoparticles, for instance in metamaterials, will rely heavily on the ability to control the large scale spatial arrangement of anisotropic nanoparticles. ${ }^{6}$ A partial success in this direction is being achieved by fabrication of anisotropic building blocks which already have embedded the information for programmed assembly, for example, topological control in the chemical composition or in the surface functionalization of each individual nanocrystal. ${ }^{5}$

Nanorods are among the most studied examples of anisotropic nanoparticles. Liquid-crystalline-like ordering in

*To whom correspondence should be addressed, liberato.manna@iit.it.

${ }^{\perp}$ Present address: Department of Chemistry, University of Chicago, Chicago, IL 60637 .

Received for review: 11/26/2009

Published on Web: 01/27/2010 both smectic and nematic phases was reported for rodshaped CdSe nanocrystals, ${ }^{7,8}$ and self-organization of CdSe nanorods into 3D superlattices was observed by destabilization of colloidal solutions upon slow diffusion of a nonsolvent. ${ }^{9}$ More generally, colloidal nanorods of various materials have been aligned in both vertically ${ }^{10-14}$ and laterally ordered arrays ${ }^{9,15}$ using a wide variety of techniques, which exploited inter-rod van der Waals or magnetic forces, applied electric fields, and substrate templating effects. ${ }^{16-19}$ The study of anisotropic nanorods for self-assembly has reached the point where it is possible to grow mono- or multilayers of rods oriented vertically or laterally with respect to the substrate or to grow micrometer-sized multilayered colloidal crystals from solution. The assembly process in the above-mentioned cases is still hard to control though, and is often the result of time-consuming, trial and error experimental work.

A possible approach to simplify and better manipulate the assembly of anisotropic particles could be their preorganization in clusters of ordered particles directly in solution followed by further building of mono- or multilayered films by convenient deposition techniques of such clusters. Various types of interparticle interactions could be exploited for the assembly, among which depletion attraction stands out. ${ }^{1,2,20,21}$ This type of interaction arises when macromolecules are added to a stable colloidal solution of nanoparticles with which they do not interact (hence they are "nonadsorbing"), but instead they have a high solubility in the solvent. The notion of depletion attraction was first introduced by Asakura and Oosawa, ${ }^{20}$ who devised a simple 
conceptual experiment, in which two large and parallel plates of a given area are immersed in a solution of spherical (rigid) macromolecules. When the distance between the plates is smaller than the diameter of the macromolecules, no macromolecule can occupy the region between the plates, which becomes a phase of pure solvent. An osmotic pressure then develops because the system tries to eliminate this region of pure solvent, and which manifests itself by an inward force (hence a "depletion force") that pushes the plates toward each other. In a solution of colloidal nanoparticles, whenever wandering nanoparticles approach each other in the liquid, a mutual attraction between them is created due to the evolution of osmotic pressure in the volume region between the particles, which cannot be occupied by the macromolecules, and hence is also a region of "pure solvent". The term "macromolecule", or "additive", as we shall call it henceforth, refers broadly to any species that is capable of eliciting such attractive interactions (purely entropic by nature) between colloidal particles. ${ }^{1,2,20,21}$ The concentration and relative size of the additive as well as the size, shape, and concentration of the particles are parameters which affect the strength of depletion interaction and which govern phase transitions in colloids. ${ }^{3}$ For decades the simple and general idea of depletion attraction was found to be responsible (or partially at least) for various phenomena, such as protein crystallization, self-assembly of viruses, and phase separation in colloids. ${ }^{22,23}$

Recent computer simulations have predicted that monolayer clusters of hexagonally close-packed rods tend to form via depletion attraction in mixtures of rods and nonadsorbing molecules of certain relative sizes. ${ }^{24-26}$ At present both theoretical modeling and simulations of rod-shaped colloids are much more advanced than experiments, mainly because the rods tested in experiments had large shape polydispersities (typically reported standard deviations in aspect ratio were above $20 \%$ ). Our recent synthetic approach to II-VI semiconductor colloidal CdS, CdSe, and heterostructured CdSe/CdS nanorods yielded samples with much narrower distributions of shapes, with polydispersities lower than $5 \%$, without any postsynthesis shape- or size-selective purification. ${ }^{12}$ These nanorods, which are coated with phosphonate and trioctylphosphine oxide (TOPO) molecules (hence they are hydrophobic), are suitable model systems for experimental testing of nanorod self-organization in solution by depletion attraction.

The starting samples in our experiments were stable and transparent colloidal solutions of nanorods in a series of organic solvents that are a good dispersant medium for the rods (we tested positively toluene, benzene, cyclohexane, and chloroform). The assembly of the nanorods in solution was triggered at room temperature by addition of a given amount of additive, which could be chosen among a wide range of molecules. To cite some examples, the formation of nanorod assemblies was observed when adding any of the following species: various long chain fatty acids and amines, the liquid crystal 4'-n-pentyl-4-cyanobiphenyl (5CB), or various polymers such as polystyrene, poly(ethylene glycol) methacrylate (PEG-MA, average $M_{\mathrm{n}} \approx 526$ ), poly(methyl methacrylate), and their copolymers. Our discussion here will be focused on the solution-phase assembly of core-shell CdSe/CdS nanorods induced by oleic acid (OA) and PEG-MA.

In solution assembly could be monitored visually by the appearance of cloudiness in an initially clear solution of nanorods in toluene (Figure $1 \mathrm{a}$ ), when the concentration of the additive exceeded a certain threshold. Confocal fluorescence microscopy imaging performed both on the starting solution and on the cloudy one revealed the presence of large fluorescent aggregates in the cloudy solution, while a homogeneously distributed fluorescence signal was observed in the starting solution (Figure 1b,c). The variation in the transparency of the solution after introducing the additive was gradual and yielded a turbid and slightly iridescent colloid which slowly precipitated in time but which could be redispersed easily by mild shaking. Depending on the exact conditions (as described below), the transition from a transparent solution to a turbid one might take from 30 min up to several hours. An intuitive guess is that this variation in turbidity is related to the formation of aggregates and to their evolution in time. To describe it more quantitatively, we used dynamic light scattering (DLS). DLS is more convenient for such characterization than other tools, e.g., low-resolution TEM of aliquots or UV-vis spectroscopy, because DLS is much faster and gives enough information for a qualitative description and comparison of the aggregation process under different experimental conditions (rods or additive concentration, type of solvent, etc.). The use of UV-vis spectroscopy to quantify variations in the optical density is on the other hand not practical in the case of CdSe/ CdS core-shell nanorods, due to their large absorption cross section. Such measurements would be possible only for diluted solutions of rods, which is not the case in this study (the typical concentration of nanorods was in a range of $10^{-6}$ M nanorods in solution, see below).

DLS data demonstrated that the average scattering intensity was generally higher, hence indicative of bigger aggregates, when either the concentration of additive or that of nanorods was increased (Figure 1e, see also ref 27), as indeed either factor enhances the attractive interactions between nanorods. In general, the concentration threshold of additive required to trigger assembly decreased with increasing molecular weight of the additive. For example, in a solution of $10^{-6} \mathrm{M}$ nanorods, this was $0.5 \mathrm{M}$ for oleic acid (OA) and 0.4 M for PEG-MA (see Figure 1 and ref 27). These observations go along well with predictions both from theory and from reported simulations (see ref 3 and references therein). The additive molecules are highly miscible with the solvent; hence they literally draw solvent away from the nanorods, causing the emergence of depletion forces 

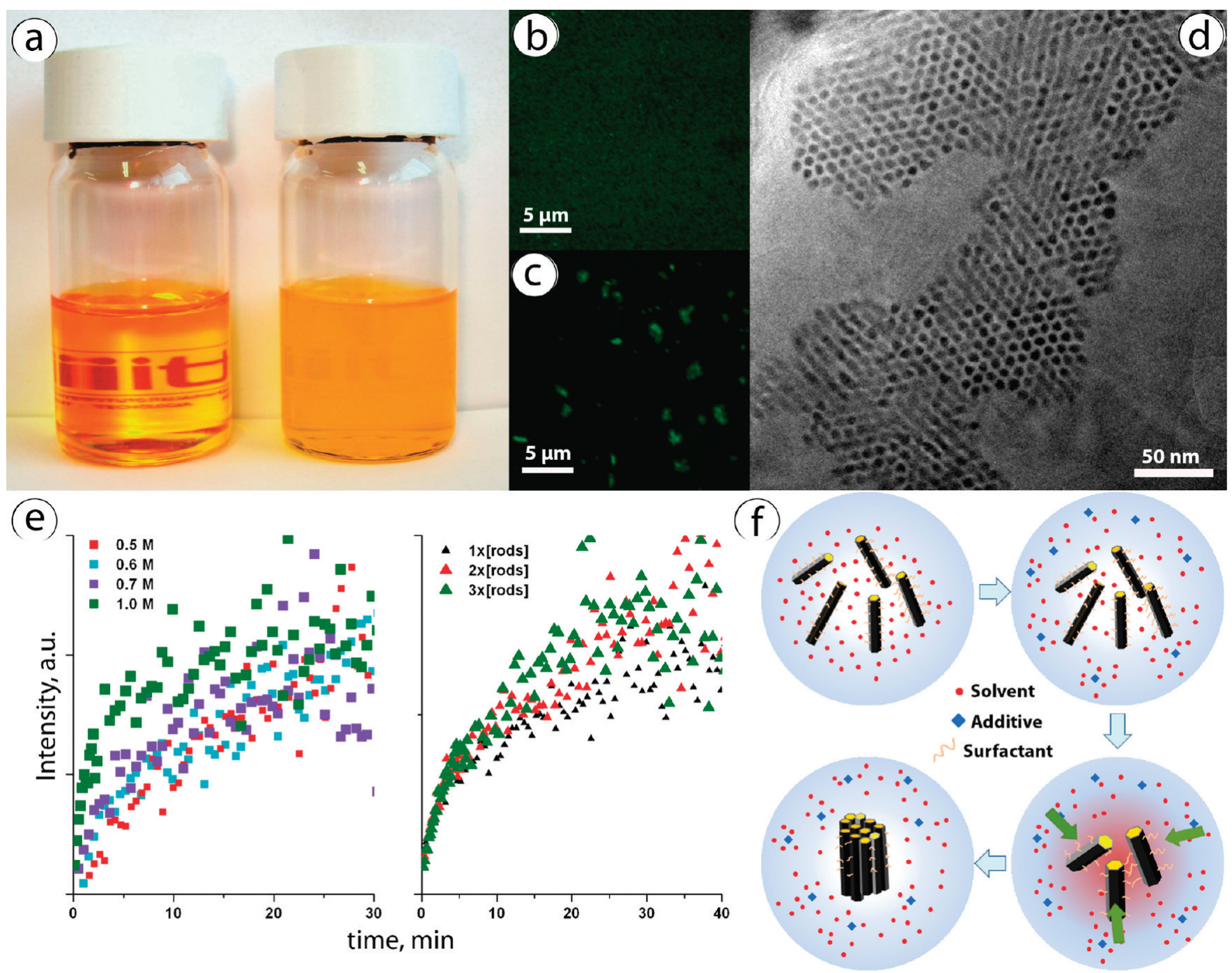

FIGURE 1. In solution assembly of colloidal nanorods. (a) Visual observation of the same solution before (left vial) and after (right vial) the assembly was triggered by addition of oleic acid. (b, c) Confocal fluorescence microscopy images of solutions placed between glass slides, before and after addition of oleic acid, respectively. (d) Cryo-TEM image of the solution after addition of oleic acid. (e) Growth evolution of the assemblies in solution, monitored by the increase of average light scattering intensity. The left graph reports a series of experiments in which the concentration of $\mathrm{OA}$ in solution was varied (from 0.5 to $1.0 \mathrm{M}$ ) while the concentration of nanorods was kept constant at $10^{-6} \mathrm{M}$. In the right graph instead the concentration of nanorods was varied (from $10^{-6}$ to $3 \times 10^{-6} \mathrm{M}$ ) while the concentration of OA was kept constant at 0.5 M. (f) A sketch of the assembly mechanism.

that induce nanorods aggregation, as schematically depicted in Figure 1f.

Cryo-TEM observations on a film of a frozen toluene solution containing nanorods and additive molecules indicated the presence of isolated 2D sheets $(50-200 \mathrm{~nm}$ in diameter) made of monolayers of hexagonally ordered rods (Figure $1 \mathrm{~d}$ and ref 27), along with the occasional presence of micrometer-sized multilayered structures. This demonstrates that hexagonal ordering of nanorods takes place already in solution and that monolayers are the predominant type of assembly, in accordance with recent calculations. ${ }^{25}$ Similar "bundling" of nanorods has been reported recently by other groups. ${ }^{28,29}$ However, it was interpreted as being driven by interlacing of the alkyl chains of the surfactants bound to the nanocrystals, at increasing concentrations of nanorods under solvent evaporation, ${ }^{28}$ or as driven by a variation in the solvent quality for the bifunctional ligands coating the rods. ${ }^{29}$ Other recent works illustrated how factors such as hydrophobic and hydrophilic interactions, or the $\mathrm{pH}$-dependent balance between electrostatic repulsive forces and attractive van der Waals forces, can lead to the formation of "superparticles" of close-packed nanorods in water but did not appear to involve depletion forces. ${ }^{30-32}$

Deposition of the cloudy solution on a variety of substrates by dipping or drop-casting, followed by solvent evaporation, yielded micrometer-sized islands of hexagonally packed nanorods arrays that were aligned vertically with respect to the substrate, irrespective of both deposition technique and surface treatment of the substrate (see Figure $2 \mathrm{a}$ as an example and ref 27 for a more in-depth coverage). 


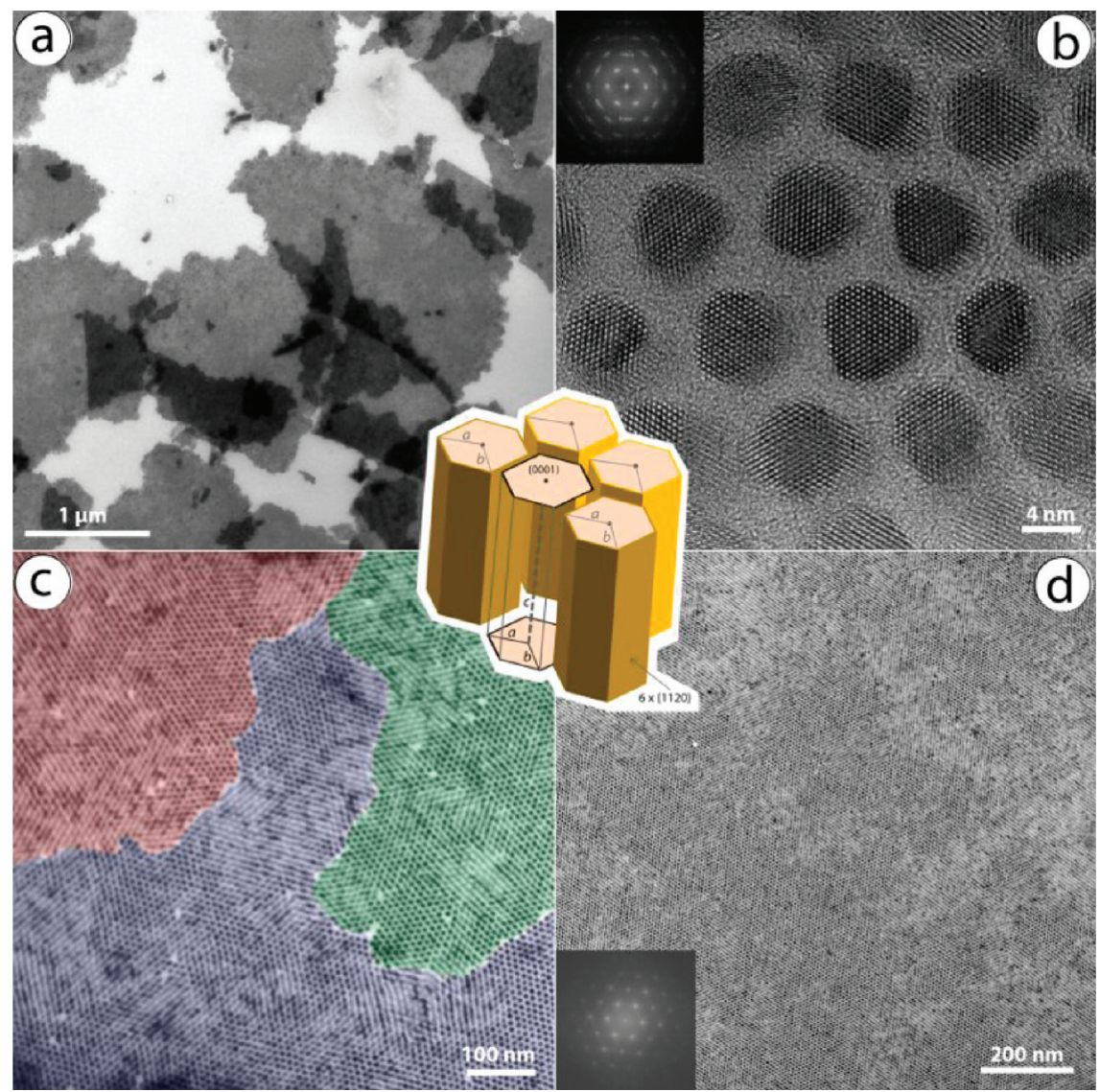

FIGURE 2. (a) Monolayer-thick sheets of vertically assembled CdSe/CdS nanorods seen under TEM. (b) High-resolution TEM image showing the mutual crystallographic alignment of the wurtzite CdSe/CdS nanorods. In the image the bright spots are columns of CdS. The inset displays the corresponding Fourier transform (FT) showing atomic spacings. (c) Area inside a monolayer sheet formed in solution showing domains of differently oriented assemblies. (d) Area of rods assembled vertically on the water surface and fished on a TEM grid shows no defects, which is confirmed by the FT showing superlattice spacings (inset). The central sketch depicts the packing of rods in the assembly.

High-resolution TEM analysis (Figure 2b) gave more insight into the assembly of nanorods in these clusters. The rods have wurtzite crystal structure and in each cluster they are aligned along their $c$-axis, which always coincides with the length direction of the rods. They have a hexagonal shape in cross section, with six $\{11 \overline{2} 0\}$ side facets while $\{10 \overline{1} 0\}$ facets are absent. This is consistent with recent surface energy and structure calculations, when surface stresses are taken into account. ${ }^{33} \mathrm{~A}$ close inspection indicates that the atomic planes of the different rods are in the same orientation despite the spacing between the rods, and therefore the crystal lattices of the rods are mutually aligned in three dimensions. This is also clear from the Fourier transform (FT) spectrum shown in the inset of Figure $2 \mathrm{~b}$. This ordering can be due to dipole interactions or favorable facet-to-facet alignment or it can be directed by interdigitation of the capping molecules. Considering that the $\{11 \overline{2} 0\}$ facets are predicted to be nonpolar, ${ }^{33}$ the most plausible causes for the mutual crystallographic orientation are then interdigitation of the ligands and the related most effective packing of hexagonal prisms (Figure 2, central inset).

In all cases, we found that there is a distinct orientation relationship between the superordering and the crystal- lographic alignment: the rods are facing each other with their $\{11 \overline{2} 0\}$ facets, and therefore the hexagonal superlattice and the hexagonal atomic lattices are rotated with respect to each other by $30^{\circ}$. Consequently, the alignment of the crystal structures extends as far as the hexagonal superordering. Numerous low-resolution TEM observations point to a multidomain structure of micrometer-sized sheets of the solution assembled rods (see Figure 2c for instance). The presence of grain boundaries suggests that larger monolayer sheets could have formed either by aggregation of small monolayer sheets nucleated in the initial stages of assembly in solution (although it is hard to imagine that smaller domains would fit perfectly to each other with few defects at the grain boundaries) or, more plausibly, by defective growth of a single, smaller monolayer. For comparison, large monolayers of rods assembled at a water/toluene interface and fished after toluene evaporation (following a previously published procedure by our group ${ }^{12}$ ), were instead single superstructures and only rarely they were mosaics made of smaller domains (see Figure $2 d$, and inset of superlattice's FT), which is indicative of a different assembly mechanism of the nanorods when an interface is present. 

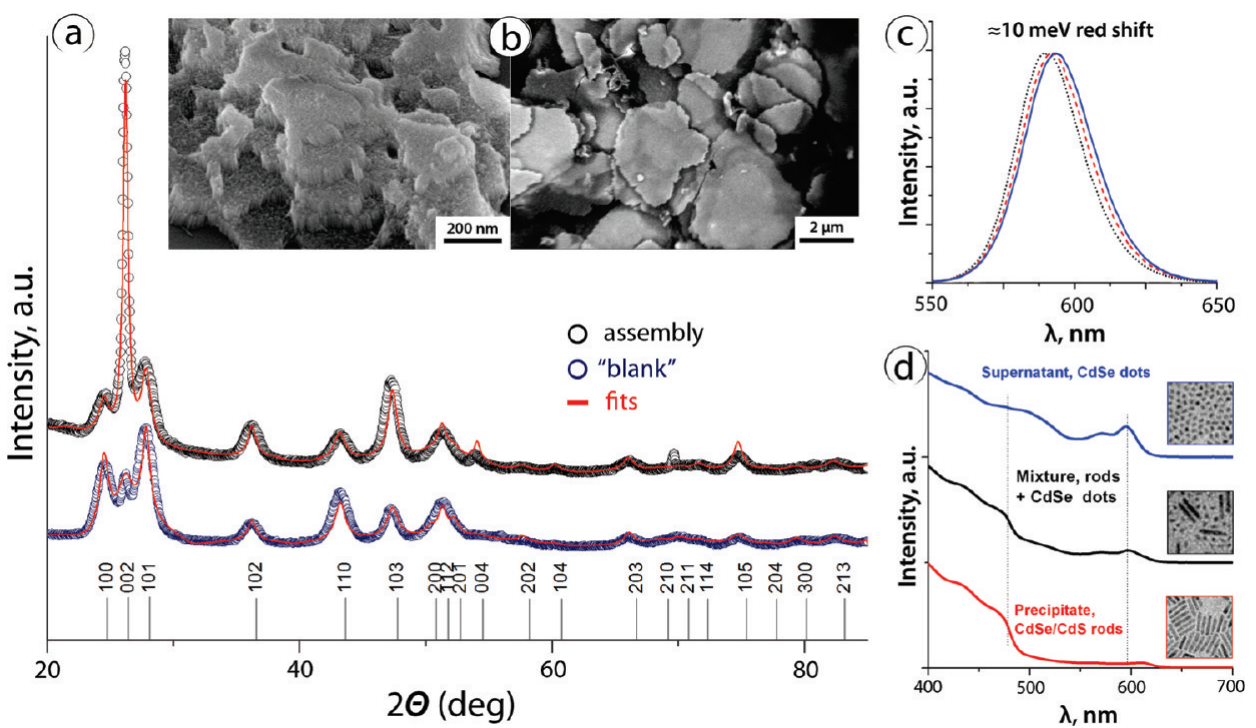

FIGURE 3. (a) Experimental XRD patterns from films of assembled rods (black circles, "assembly") drop-cast from a suspension in 1-butanol, and from films of unassembled rods (blue circles, "blank") drop-cast from a toluene solution. In both cases the substrate was $\mathrm{Si} / \mathrm{SiO}_{2}$. The best fitted XRD patterns from both samples were calculated by introducing in the modeling both anisotropic peak broadening (shape effect) and preferred orientations (PO). The diffraction pattern of the "blank" was described by two POs (along the $h=100$ and $h=002$ ) in the fractions corresponding to $66 \%$ and $34 \%$, respectively, while the pattern of "assembly" was described by $100 \%$ of PO along the $h=002$ ( $c$-axis of wurtzite). (b) SEM images of the films of assembled rods, at two different magnifications. (c) PL spectra of nonassembled rods (black dotted line) and rods assembled by addition of oleic acid (red dashed line) show $10 \mathrm{meV}$ red shift, corresponding to the increased rod-to-rod coupling in assemblies. Transfer of the assemblies into nonsolvent (ethanol, blue solid line) does not affect PL spectrum further (see ref 27 for a more complete set of PL data). (d) Shape separation in a binary mixture of rods and CdSe dots as monitored by optical absorption and TEM.

These two-dimensional sheets of assembled rods were controllable in size and robust. Although their self-organization resulted in irregularly shaped sheets, their average lateral size was tunable from hundreds of nanometers up to $1-2 \mu \mathrm{m}$, depending on the concentration of rods and additive, time of aging, and stirring. If the solution was not stirred after the assemblies were formed, micrometer-sized sheets precipitated at the bottom of the vial due to gravity. The supernatant, which was a mixture of solvent and additive, could then be exchanged with a pure solvent. If this solvent was of the type in which the rods are soluble (see above), the assemblies were completely dissolved in it, yielding an optically clear and stable solution of isolated nanorods, and no change in morphology or in optical properties with respect to the starting nanorod solution could be observed.

The addition of a liquid in which the rods were not soluble, hence a "nonsolvent" (like 1-butanol, see ref 27 for a list of "nonsolvents" tested) yielded instead a suspension of sheets. The original sheets were not disrupted, neither their sizes nor shapes were modified significantly. Comparison of low-resolution TEM images of assemblies did not reveal any remarkable changes in inter-rod distances after solvent exchange, regardless of the type of additive used to induce the assembly, which can be attributed to the additive molecules not being able to intercalate between nanorods in the assemblies. ${ }^{27}$ The "nonsolvent" in this case prevented the disassembly of rods, due to their poor solubility in it, while it helped to remove the excess of additive that contaminated the assemblies. Drop-casting of these suspensions in a nonsolvent onto a silicon substrate yielded multilayered films with a remarkably high degree of vertical nanorod ordering with respect to the substrate as revealed by X-ray diffraction and scanning electron microscopy (top XRD pattern of panels a and b of Figure 3). In contrast, dropcasting of a solution of unassembled rods in toluene yielded films in which the nanorods were preferentially laying flat with respect to the substrate (bottom XRD pattern of Figure 3a). Photoluminescence (PL) spectra of the rods assembled by addition of oleic acid and of the same assemblies transferred into a nonsolvent showed in both cases a $10 \mathrm{meV}$ red shift with respect to the PL nonassembled rods, which could be due to the increased rod-to-rod coupling in assemblies (Figure $3 \mathrm{c}$ and ref 27), in analogy with previous works on nanocrystal superstructures. ${ }^{34}$

In principle, chemical reactions can be carried out directly in solution on these suspensions of nanorod assemblies. As an example, their exposure to a diluted solution of hydrazine effectively exchanged the surfactants that coated the surface of the nanorods with hydrazine molecules and led to a considerable reduction in the inter-rod distances in the floating sheets. ${ }^{27}$ To the best of our knowledge, this is the first example of chemical treatment carried out on a "floating", unsupported assembly of nanoparticles. This approach has the potential to resolve the problem of cracking in chemically treated films of assembled nanoparticles on substrates: ${ }^{27}$ in the present case each sheet can easily accommodate for the compressive strain developed upon 
surfactant exchange without cracking, after which larger sheets can still be built by further assembly of these surfactant exchanged sheets.

It is important to stress here that many well-known surfactants that are widely used for the synthesis and processing of colloidal nanoparticles (oleic and stearic acids, oleylamine, hexadecylamine, etc.) are among the additives which were found to induce depletion attraction between nanorods in solution. This led us to the conclusion that even incomplete washing of the as-prepared nanocrystals from the excess of surfactants typically used in the synthesis may dramatically affect the self-assembly process. If an excess of free surfactant is present, solvent evaporation implies an increase in the relative free surfactant concentration at the late stages of drying. Therefore there is a possibility that depletion attraction forces may significantly contribute to the self-assembly process. This aspect was already highlighted in a recent work, in which the critical role of the excess of free oleic acid was noted in the formation of binary nanocrystal superlattices and in which the possible contribution of depletion attraction forces was considered. ${ }^{35}$

In addition to providing a simple and effective means for fabricating ordered assemblies of nanorods, depletion attraction forces can be employed for the shape-selective precipitation from binary or more complex nanoparticle mixtures. The magnitude of depletion forces is strongly related to the shape and size of the particles and is significantly larger for rods than for spheres, due to the higher gain in overlapped excluded volume in rods, which decreases the free energy of the system. ${ }^{2}$ As a straightforward consequence, in a binary mixture of rods and spheres of similar diameters, rods should start assembling and then precipitate upon gradual increase in additive concentration. As a proof of principle, CdSe/CdS nanorods $(6 \mathrm{~nm}$ diameter $\times 24 \mathrm{~nm}$ length) were quantitatively precipitated from a binary mixture that contained additionally either CdTe or CdSe nanocrystal spheres (4-5 $\mathrm{nm}$ in diameter), as demonstrated by absorption spectroscopy and TEM analysis (Figure $3 d$, see also ref 27). Quite interestingly, even large additions of additive molecules could not precipitate the spheres, indicating that for the present mixture of nanocrystals the depletion forces among the spheres were not able to overcome a threshold for nanoparticle aggregation. This is different from the standard size/shape selective precipitation procedures which employ a nonsolvent (i.e., methanol), since careful additions need to be performed in those cases in order not to precipitate all the particles present in solution. Preferential precipitation of gold rods in mixtures of rods, spheres and platelets by using an excess of surfactants in water was previously observed by Jana ${ }^{36}$ and the complete rods separation required several steps in that study, while in our case the separation was quantitative after the first centrifugation. In view of these results, shape-selective precipitation mediated by depletion forces represents a more robust approach for shape-sorting of colloidal nanoparticles, with the ad- ditional appealing feature of being environmentally friendlier, as it can avoid the use of large amounts of toxic solvents.

In conclusion, additive-induced depletion attraction between colloidal semiconductor nanorods was exploited to nucleate micrometer-sized sheets of vertically assembled rods directly in solution. Once formed, the assemblies could be used as building blocks for constructing larger one- or multilayer sheets of ordered rods, and additionally these sheets could be chemically modified directly in solution. The versatility of handling and processability of these suspensions appears comparable to that of graphene sheets. ${ }^{37}$ Moreover, shape-selective separation of nanorods from dots in a binary mixture was demonstrated. This approach offers a new tool for the effective manipulation of anisotropic nanoparticles and will help to reach the goal of fabricating new materials and devices based on ordered assemblies of shape-controlled nanostructures.

Acknowledgment. The authors acknowledge financial support from the European Union, through the FP6-3I program (ref. 026019 ESTEEM) and the FP7 program (ERC Starting Grant NANO-ARCH, Contract Number 240111). Marco Scotto is thanked for his technical help on confocal microscopy experiments.

Supporting Information Available. Detailed description of the synthesis of nanorods and of their assembly in solution, transfer to various substrates; transfer of nanorod assemblies to nonsolvents and details of hydrazine treatment; description of the various characterization approaches; additional data on dynamic light scattering characterization; additional confocal, TEM, and SEM images of the assemblies; photoluminescence spectra of CdSe/CdS nanorods dispersed in toluene, of nanorod assemblies in toluene, and of assemblies transferred to various nonsolvents; wide-field lowresolution TEM images of binary mixtures of $\mathrm{CdSe} / \mathrm{CdS}$ nanorods and of CdSe dots before shape-selective precipitation with oleic acid and TEM images of corresponding fractions after addition of oleic acid. This material is available free of charge via the Internet at http://pubs.acs.org.

\section{REFERENCES AND NOTES}

(1) Min, Y. J.; Akbulut, M.; Kristiansen, K.; Golan, Y.; Israelachvili, J. Nat. Mater. 2008, 7 (7), 527-538.

(2) Bishop, K. J. M.; Wilmer, C. E.; Soh, S.; Grzybowski, B. A. Small 2009, 5 (14), 1600-1630

(3) Anderson, V. J.; Lekkerkerker, H. N. W. Nature 2002, 416 (6883), $811-815$.

(4) Shevchenko, E. V.; Talapin, D. V.; Kotov, N. A.; O’Brien, S.; Murray, C. B. Nature 2006, 439 (7072), 55-59.

(5) Glotzer, S. C.; Solomon, M. J. Nat. Mater. 2007, 6 (8), 557-562.

(6) Stebe, K. J.; Lewandowski, E.; Ghosh, M. Science 2009, 325 (5937), $159-160$

(7) Li, L. S.; Alivisatos, A. P. Adv. Mater. 2003, 15 (5), 408-411.

(8) Li, L. S.; Walda, J.; Manna, L.; Alivisatos, A. P. Nano Lett. 2002, 2 (6), 557-560

(9) Talapin, D. V.; Shevchenko, E. V.; Murray, C. B.; Kornowski, A.; Forster, S.; Weller, H. J. Am. Chem. Soc. 2004, 126 (40), $12984-$ 12988.

(10) Ahmed, S.; Ryan, K. M. Nano Lett. 2007, 7 (8), 2480-2485.

(11) Ryan, K. M.; Mastroianni, A.; Stancil, K. A.; Liu, H. T.; Alivisatos, A. P. Nano Lett. 2006, 6 (7), 1479-1482. 
(12) Carbone, L.; Nobile, C.; De Giorgi, M.; Sala, F. D.; Morello, G.; Pompa, P.; Hytch, M.; Snoeck, E.; Fiore, A.; Franchini, I. R.; Nadasan, M.; Silvestre, A. F.; Chiodo, L.; Kudera, S.; Cingolani, R.; Krahne, R.; Manna, L. Nano Lett. 2007, 7 (10), 2942-2950.

(13) Guerrero-Martínez, A.; Pérez-Juste, J.; Carbó-Argibay, E.; Tardajos, G.; Liz-Marzán, L. M. Angew. Chem., Int. Ed. 2010, 121 (50), 96489652.

(14) Baker, J. L.; Widmer-Cooper, A.; Toney, M. F.; Geissler, P. L.; Alivisatos, A. P. Nano Lett. 2010, 10, 195-201.

(15) Sun, B. Q.; Sirringhaus, H. J. Am. Chem. Soc. 2006, 128 (50), $16231-16237$

(16) Wetz, F.; Soulantica, K.; Respaud, M.; Falqui, A.; Chaudret, B. Mater. Sci. Eng., C 2007, 27 (5-8), 1162-1166

(17) Talapin, D. V.; Shevchenko, E. V.; Murray, C. B.; Titov, A. V.; Kral P. Nano Lett. 2007, 7 (5), 1213-1219.

(18) Querner, C.; Fischbein, M. D.; Heiney, P. A.; Drndic, M. Adv Mater. 2008, 20 (12), 2308-2314.

(19) Gupta, S.; Zhang, Q. L.; Emrick, T.; Russell, T. P. Nano Lett. 2006 6 (9), 2066-2069.

(20) Asakura, S.; Oosawa, F. J. Chem. Phys. 1954, 22, 1255-1256

(21) Asakura, S.; Oosawa, F. J. Polym. Sci. 1958, 33, 183-192.

(22) Cohen, S. S. J. Biol. Chem. 1942, 144, 353-363.

(23) Dogic, Z.; Fraden, S., Phase Behavior of Rod-Like Viruses and Virus-Sphere Mixtures. In Soft Matter; Gompper, G., Schick, M., Eds.; Wiley: New York, 2007

(24) Savenko, S. V.; Dijkstra, M. J. Chem. Phys. 2006, 124, 234902.
(25) Cuetos, A.; van Roij, R.; Dijkstra, M. Soft Matter 2008, 4 (4), 757 767 .

(26) Patti, A.; Dijkstra, M. Phys. Rev. Lett. 2009, 102 (12), 128301.

(27) Additional details on materials and methods are available in the Supporting Information.

(28) Kang, C. C.; Lai, C. W.; Peng, H. C.; Shyue, J. J.; Chou, P. T. Acs Nano 2008, 2 (4), 750-756

(29) Zhao, N.; Liu, K.; Greener, J.; Nie, Z. H.; Kumacheva, E. Nano Lett. 2009, 9 (8), 3077-3081

(30) Zhuang, J. Q.; Shaller, A. D.; Lynch, J.; Wu, H. M.; Chen, O.; Li, A. D. Q.; Cao, Y. C.J. Am. Chem. Soc. 2009, 131 (17), 6084-6085.

(31) Sanyal, A.; Bala, T.; Ahmed, S.; Singh, Ajay; Piterina, A. V.; McGloughlin, T. M.; Laffir, F. R.; Ryan, K. M.J. Mater. Chem. 2009, 42, $6421-6423$.

(32) Fang, X. L.; Li, Y.; Chen, C.; Kuang, Q.; Gao, X. Z.; Xie, Z. X.; Xie, S. Y.; Huang, R. B.; Zheng, L. S. Langmuir 2009, DOI: $10.1021 /$ la902765p.

(33) Barnard, A. S.; Xu, H. J. Phys. Chem. C. 2007, 111 (49), $18112-$ 18117.

(34) Schmidt, G., Nanoparticles: from theory to applications, 4th ed.; Wiley: Weinheim, 2006

(35) Smith, D. K.; Goodfellow, B.; Smilgies, D. M.; Korgel, B. A.J. Am. Chem. Soc. 2009, 131 (9), 3281-3290.

(36) Jana, N. R. ChemComm. 2003, 15, 1950-1951.

(37) Park, S.; Ruoff, R. S. Nat. Nanotechnol. 2009, 4 (4), 217-224. 